Please do not remove this page

RMIT

UNIVERSITY

\title{
Investigation of two-solvent grinding-assisted liquid phase exfoliation of layered Mos2
}

Nguyen, Emily; Carey, Benjamin; Daeneke, Torben; Ou, Jianzhen; Latham, Kay; Zhuiykov, Serge; Kalantar Zadeh, Kourosh

https://researchrepository.rmit.edu.au/esploro/outputs/9921862471401341/filesAndLinks?institution=61RMIT_INST\&index=null

Nguyen, E., Carey, B., Daeneke, T., Ou, J., Latham, K., Zhuiykov, S., \& Kalantar Zadeh, K. (2015).

Investigation of two-solvent grinding-assisted liquid phase exfoliation of layered MoS2. Chemistry of Materials, 27(1), 53-59. https://doi.org/10.1021/cm502915f

Document Version: Accepted Manuscript

Published Version: https://doi.org/10.1021/cm502915f

Repository homepage: https://researchrepository.rmit.edu.au

(c) 2014 American Chemical Society

Downloaded On 2023/04/26 20:52:39 +1000

Please do not remove this page 
Thank you for downloading this document from the RMIT Research Repository.

The RMIT Research Repository is an open access database showcasing the research outputs of RMIT University researchers.

RMIT Research Repository: http://researchbank.rmit.edu.au/

\author{
Citation: \\ Nguyen, E, Carey, B, Daeneke, T, Ou, J, Latham, K, Zhuiykov, S and Kalantar \\ Zadeh, K 2015, 'Investigation of two-solvent grinding-assisted liquid phase \\ exfoliation of layered MoS2', Chemistry of Materials, vol. 27, no. 1, pp. 53-59.
}

See this record in the RMIT Research Repository at:

https://researchbank.rmit.edu.au/view/rmit:32757

Version: Accepted Manuscript

Copyright Statement:

(c) 2014 American Chemical Society

Link to Published Version:

http://dx.doi.org/10.1021/cm502915f 


\title{
Investigation of two-solvent grinding assisted liquid phase exfoliation of layered $\mathrm{MoS}_{2}$
}

\author{
Emily P. Nguyen ${ }^{1,2 *}$, Benjamin J. Carey ${ }^{1,2}$, Torben Daeneke ${ }^{1}$, Jian Z. Ou ${ }^{1}$, Kay Latham ${ }^{3}$, Serge \\ Zhuiykov $^{2}$ and Kourosh Kalantar-zadeh ${ }^{1 *}$
}

1. School of Electrical and Computer Engineering, RMIT University, Melbourne, Victoria, Australia

2. Division of Materials Science and Engineering, CSIRO, Highett, Victoria, Australia

3. School of Applied Science, RMIT University, Melbourne, Victoria, Australia

KEYWORDS: MoS2, exfoliation, solvent, grinding-assisted

\begin{abstract}
Grinding-assisted sonication exfoliation of stratified materials such as $\mathrm{MoS}_{2}$ is a widely used method for the preparation of their single and few layer thick flakes. This work introduces a two-solvent step approach utilizing a separate solvent during the grinding phase, while implementing ethanol during exfoliation. It is found that the grinding solvent played a critical role, determining exfoliation yield, flake dimensions and morphology, highlighting the importance of such parameters in the process. Furthermore, it is found that the commonly used N-methyl-2-pyrrolidone (NMP) lead to persistent residues on the exfoliated flakes which may alter the properties of the flakes and interfere with the development of electronic devices and other applications. A solvent residue free exfoliation method is presented herein, which may be advantageous for future studies.
\end{abstract}

\section{Introduction}

In recent years, layered semiconducting nanomaterials have gained increased attention and have been intensely studied due their unique properties. Within the layered materials family, great interest has been given to transition metal dichalcogenindes, where molybdenum disulfide $\left(\mathrm{MoS}_{2}\right)$ has gained the most attention. [1,2]

The stratified $2 \mathrm{H} \mathrm{MoS}_{2}$ crystal structure is composed of layers of Mo atoms sandwiched between $\mathrm{S}$ atoms that are covalently bonded to form a trigonal prismatic arrangement. [3-5] In the bulk crystal, many of these layers are stacked in registry on top of each other via Van der Waals forces. [1, 2, 6] Bulk $\mathrm{MoS}_{2}$ has a band gap of $\sim 1.2$ $\mathrm{eV}$, however, upon reduction of the number of layers towards a single monolayer, the dominant band gap increases to $\sim 1.8 \mathrm{eV}$. [1, 4, 6-8] Simultaneously, the indirect bandgap transitions to a direct bandgap leading to the rise of photoluminescence (PL), which is not observed in the bulk material. $[5,7,9,10] 2 \mathrm{H} \mathrm{MoS}_{2}$ exfoliated into just a few atomic layer thickness has found tremendous popularity in field effect transistors with impressively large ON/OFF ratios.[11] Further applications have been demonstrated in energy storage [12, 13], catalysis [6, 14], and sensing [15].

While mechanical exfoliation, as well as many physical and chemical vapour deposition techniques, have been developed for producing high quality two dimensional (2D) $\mathrm{MoS}_{2}$ crystals, such methods are generally suited for electronic device fabrication.[16] Solution processed films are of more interest for applications such as catalysis, energy storage and, to some extent, sensing. In such cases, liquid exfoliation methods resulting in high yields and quality are greatly sought after to produce thick and porous films of two dimensional flakes.

So far, liquid phase exfoliation techniques have shown to be the highest yielding technique when starting from a bulk material.[17-20] The main methods utilized are either ion intercalation or sonication assisted exfoliation. [17, 21] One of the most common ion intercalation approaches involves the intercalation of lithium ions $\left(\mathrm{Li}^{+}\right)$in the interlayer space of the bulk material, followed by an immersion of the resulting compound in water. The $\mathrm{Li}^{+}$ reacts with water to form $\mathrm{LiOH}$ and $\mathrm{H}_{2}$, which expands the interlayered space causing the bulk material to exfoliate into 2D materials.[21-23] However, it was found that the harsh conditions led to defects and the introduction of phase impurity in the synthesized 2D MoS $\mathrm{D}_{2}$ flakes, which alter the electronic and physical properties of the product. [24] Additionally, the educts used in such processes, especially n-butyl lithium, are highly flammable and explosive, posing health hazards and complicating the upscale of such processes to industrial quantities. An alternative approach uses the electrochemical intercalation of $\mathrm{Li}^{+}$, avoiding the use of dangerous chemicals. Unfortunately, this method results in low yields, thus defeating the purpose.[25]

Another liquid phase exfoliation method utilizes high intensity ultrasound waves (sonication) to exfoliate the bulk material. Thin layers are exfoliated from the layered bulk material using sonication, while dispersed in polar solvents, with and without the aid of surfactants.[21] Via centrifugation, the resultant $2 \mathrm{D}$ material is then separated into different lateral dimensions and collected. These methods are favorable, as they do not involve any chemical reactions, are not air-sensitive and generally show acceptable yields.[21] Yao et al. have reported to obtaining relatively higher yields, up to $26.7 \mathrm{mg} / \mathrm{mL}$, by incorporating grinding into their exfoliation procedure. [26]

For sonication methods the solvent selection plays an important part since physical properties such as boiling points [27], surface tension and energy, [21, 28] as well as solubility parameters [17] affect the resulting 2D material. 
There are still many challenges and unknowns when utilizing the sonication exfoliation method and further work is necessary in order to understand the process and further improving the quality of the resulting 2D $\mathrm{MoS}_{2}$ flakes.

Coleman et al. have compiled a list of many solvents and have evaluated their suitability for the sonication exfoliation of 2D materials. The most effective solvent was found to be N-methylpyrrolidone (NMP).[28] As a result, NMP is the solvent of choice used in the majority of published research. Even though good yields are obtained when using NMP, the solvent is difficult to be removed due to a relatively high boiling point. Residual NMP can often be found on the resulting $2 \mathrm{D}$ materials, even when post processing methods are used. [17] Methods such as refluxing at low temperatures proved to be ineffective and at high temperatures the material oxidises or transforms into its metallic states depending on the environment. ${ }^{\dagger}$ The complete removal of the process solvent is, however, instrumental to many applications, especially for the fabrication of novel sensors and catalysts which rely on the availability of large and pristine surface areas.

In this work, we aim to evaluate the effect of different organic solvents for the exfoliation of $\mathrm{MoS}_{2}$ using the grinding-assisted liquid phase exfoliation method. The solvents used are acetone, acetonitrile (ACN), benzene, cyclohexane (C. Hex.), hexane (Hex.), isopropanol, methanol and toluene and for compression NMP. These solvents have lower boiling points, and surface tension energies, than

that

Table 1: Boiling points and surface tension energies of the solvent used in this work[29]

\begin{tabular}{ccc}
\hline Solvent & $\begin{array}{c}\text { Surface tension } \\
\left(\mathbf{m J} / \mathbf{m}^{\mathbf{2}} \mathbf{a t} \mathbf{2 0}{ }^{\circ} \mathbf{C}\right)\end{array}$ & Boiling point $\left({ }^{\circ} \mathbf{C}\right)$ \\
\hline NMP & 40.79 & 202.0 \\
Acetone & 25.20 & 56.5 \\
Acetonitrile & 29.50 & 82.0 \\
Benzene & 28.90 & 80.1 \\
Cyclohexane & 24.95 & 80.7 \\
Hexane & 18.00 & 69.0 \\
Isopropanol & 23.00 & 82.6 \\
Methanol & 22.70 & 64.7 \\
Toluene & 28.40 & 111.0 \\
\hline
\end{tabular}

of NMP (Table 1), which should ease the removal of the process solvent from the surface of the $2 \mathrm{D} \mathrm{MoS}_{2}$ flake. Herein the investigated organic solvents are used exclusively in the grinding stage of the exfoliation. After the grinding step, the samples are dispersed into ethanol for the sonication and centrifugation stages. Profound differences are found between the samples; which highlights the importance of the grinding step and the used solvent during this process in particular that are fully discussed. Raman spectroscopy and optical characterizations are utilized to assess the presence and the effect of residues on the surface of the grinding assisted liquid phase exfoliated 2D MoS 2 .

\section{Experimental Section \\ Exfoliation of $\mathrm{MoS}_{2}$}

$2 \mathrm{~g}$ of $\mathrm{MoS}_{2}$ powder (Sigma Aldrich, $\sim 6 \mu \mathrm{m}$ ) and $0.5 \mathrm{~mL}$ of the chosen solvent were manually ground using a mortar and pestle for 30 mins. While grinding, an extra $0.5 \mathrm{~mL}$ of solvent was also added in $0.1 \mathrm{~mL}$ doses to replenish evaporated solvent. The powder was then left to dry overnight in a vacuum oven $(\sim 500 \mathrm{mmHg})$ at $60{ }^{\circ} \mathrm{C}$ to evaporate the solvent. The dried sample was then redispersed in $40 \mathrm{~mL}$ ethanol and probe sonicated (Ultrasonic with temperature controller, GEX 500) for 90 mins at $125 \mathrm{~W}(20 \mathrm{kHz})$ with stirring. Afterwards, the solution was centrifuged for $60 \mathrm{mins}$ at $4000 \mathrm{rpm}$ (Boeco, C-28A) and the supernatant was decanted. The supernatant was then centrifuged again for $30 \mathrm{mins}$ at $4000 \mathrm{rpm}$ and the supernatant, containing the nanoflakes, was collected. The two step centrifugation procedure ensured complete removal of bulk powder.

\section{Characterisations}

Raman spectra were obtained using a micro-Raman spectrometer (InVia Streamline microscope, Renishaw, $\mathrm{UK}$ ) with a $485 \mathrm{~nm}$ laser source at $1 \mathrm{~mW}$ power. $2.5 \mathrm{~mL}$ of each suspension was drop-casted onto silicon substrate (wafer) and heated at $40{ }^{\circ} \mathrm{C}$ on a hotplate to evaporate the solvent.

A scanning probe microscope (SPM/AFM) (D3100, Digital Instruments) was used to measure the surface contours and the thickness of the exfoliated flakes. Conductive AFM images were obtained using a Bruker MultiMode with an installed Peak Force TUNA module (MM8-PFTUNA for MultiMode8 AFM system, Germany). X-ray diffraction (XRD) patterns were obtained using Rigaku miniflex II.

The nanoflake suspensions were diluted further for dynamic light scattering (DLS) readings. $1 \mathrm{~mL}$ of the suspension was pipetted into a DLS tube and was diluted by adding $1 \mathrm{~mL}$ of filtrated (220 nm membrane filter) ethanol. The tube was shaken and bench bath sonicated to mix its contents prior to measuring the particle size by DLS, using an ALV Fast DLS particle sizing spectrometer. PL measurements were obtained with a spectrofluorometer (Fluoromax 4C, Horiba Scientific) using a fluorescence cuvette. UV-Vis spectra were obtained on a UV-Vis spectrometer (Cary 60, Algilent Technologies), with a standard plastic cuvette. No dilution was required for the PL and $\mathrm{UV}-\mathrm{V}$ is measurements. 
(a)
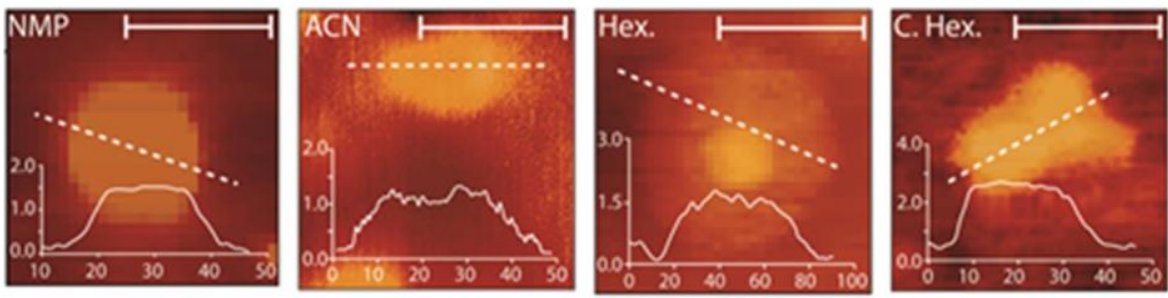

(b)
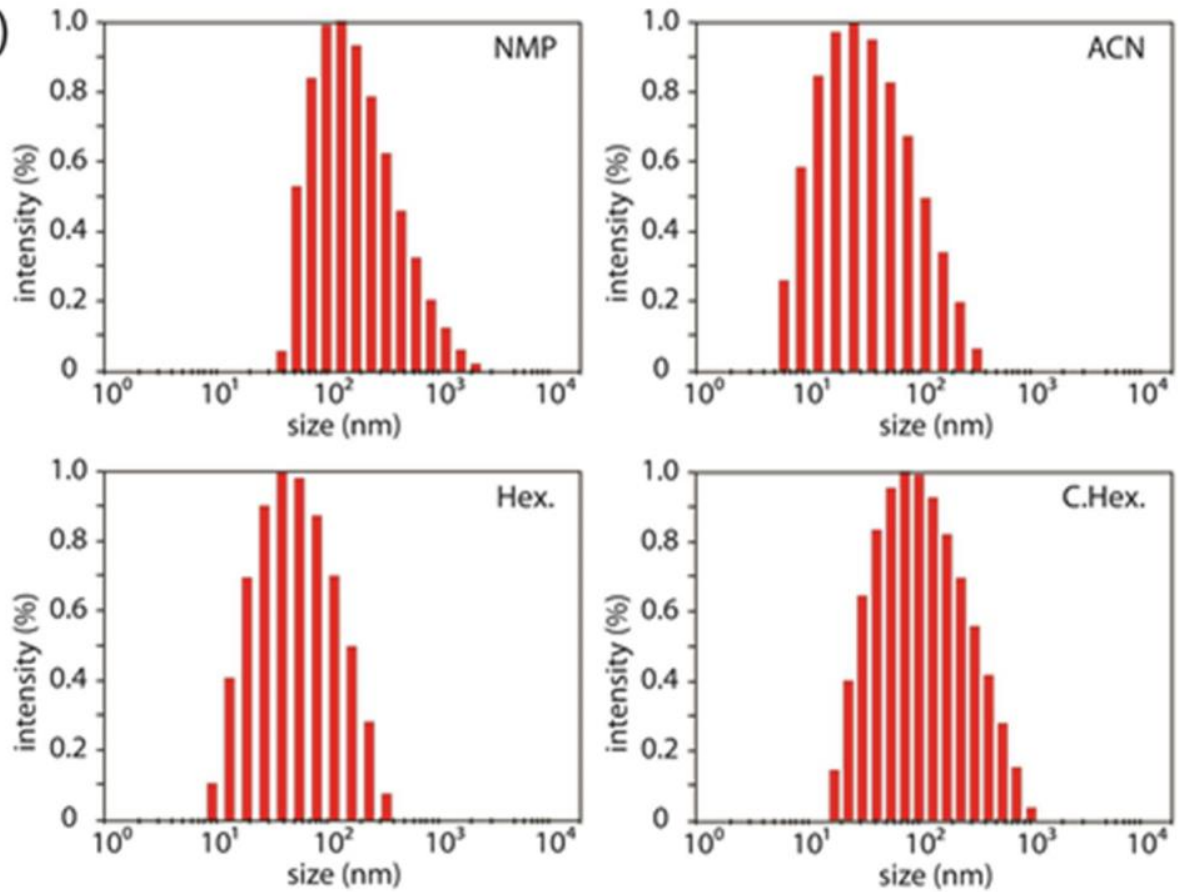

Figure 1: (a) AFM profiles (scale bar is $50 \mathrm{~nm}$ ) and (b) DLS size distribution of $\mathrm{MoS}_{2}$ when exfoliated in various solvents.

A droplet of each suspension was dropped onto a 300 mesh copper lacey carbon grid (ProSciTech, Australia). The dried samples were viewed with a Jeol 2010 transmission electron microscope (TEM) equipped with a SC600 CCD Camera (Gatan Orius).

\section{Discussion}

The suspensions of quasi 2D (Q2D) $\mathrm{MoS}_{2}$ nanoflakes (they are called Q2D as their lateral length to thickness ratios do not exceed 100, which will be shown later) were prepared using the grinding-assisted liquid phase exfoliation method, adopted from Yao et al.[26] The solvents used during the grinding procedure include NMP, ACN, Hex., C. Hex., isopropanol, methanol, acetone, benzene and toluene. Following the synthesis procedure (see Methods), isopropanol, methanol, acetone, benzene and toluene ground $\mathrm{MoS}_{2}$ did not result in measureable (the concentrations were too low to for the characterization purposes (see supporting information S1)) concentrations of Q2D $\mathrm{MoS}_{2}$ in the final ethanolic suspension.

Grinding the bulk $\mathrm{MoS}_{2}$ in NMP, ACN, Hex. and C. Hex. followed by probe sonication was found to lead to appreciable Q2D $\mathrm{MoS}_{2}$ concentrations in the final solutions. This finding highlights the importance of the grinding step and the choice of solvent during this process. After grinding in the chosen solvents, the suspensions were dried at elevated temperatures and reduced pressure for several hours in an attempt to remove any solvent residues. All samples were then redispersed, probe sonicated and centrifuged in ethanol.

The physical dimensions of the Q2D flakes were characterized by AFM imaging (Fig. 1a). AFM profiles showed that the surfaces were flat, an indication that $2 \mathrm{D}$ flakes were obtained. The exfoliated flakes produced with NMP and C. Hex. were typically 1.5 to $3.5 \mathrm{~nm}$ in height, which corresponds to 2-5 layers of $\mathrm{MoS}_{2}$ (single layer thickness: $\sim 0.7 \mathrm{~nm}[10,30])$, with lateral dimensions most frequently ranging about 20 to $200 \mathrm{~nm}$. The flakes exfoliated with ACN varied in height, ranging from 1.5 to $7 \mathrm{~nm}$ (up to 10 layers), with the majority being between 3.5 to $5 \mathrm{~nm}$ thick (5 to 7 layers) while featuring 10 to 50 $\mathrm{nm}$ lateral dimensions. Exfoliation with Hex. produced the thickest flakes measuring heights of 5.5 to $11 \mathrm{~nm}$ (up to 16 layers) and lateral dimensions of 20 to $120 \mathrm{~nm}$.

The lateral dimensions were also measured with DLS and the distribution of particle diameters were determined following Lotya et al.(Figure 1b).[31] The distribution of the particle size suggests that all samples have a high polydispersity. The median lateral dimensions for NMP, ACN, Hex. and C. Hex were 110, 30, 40 and $80 \mathrm{~nm}$, respectively. The two dimensional character of the Q2D flakes can be approximated by 

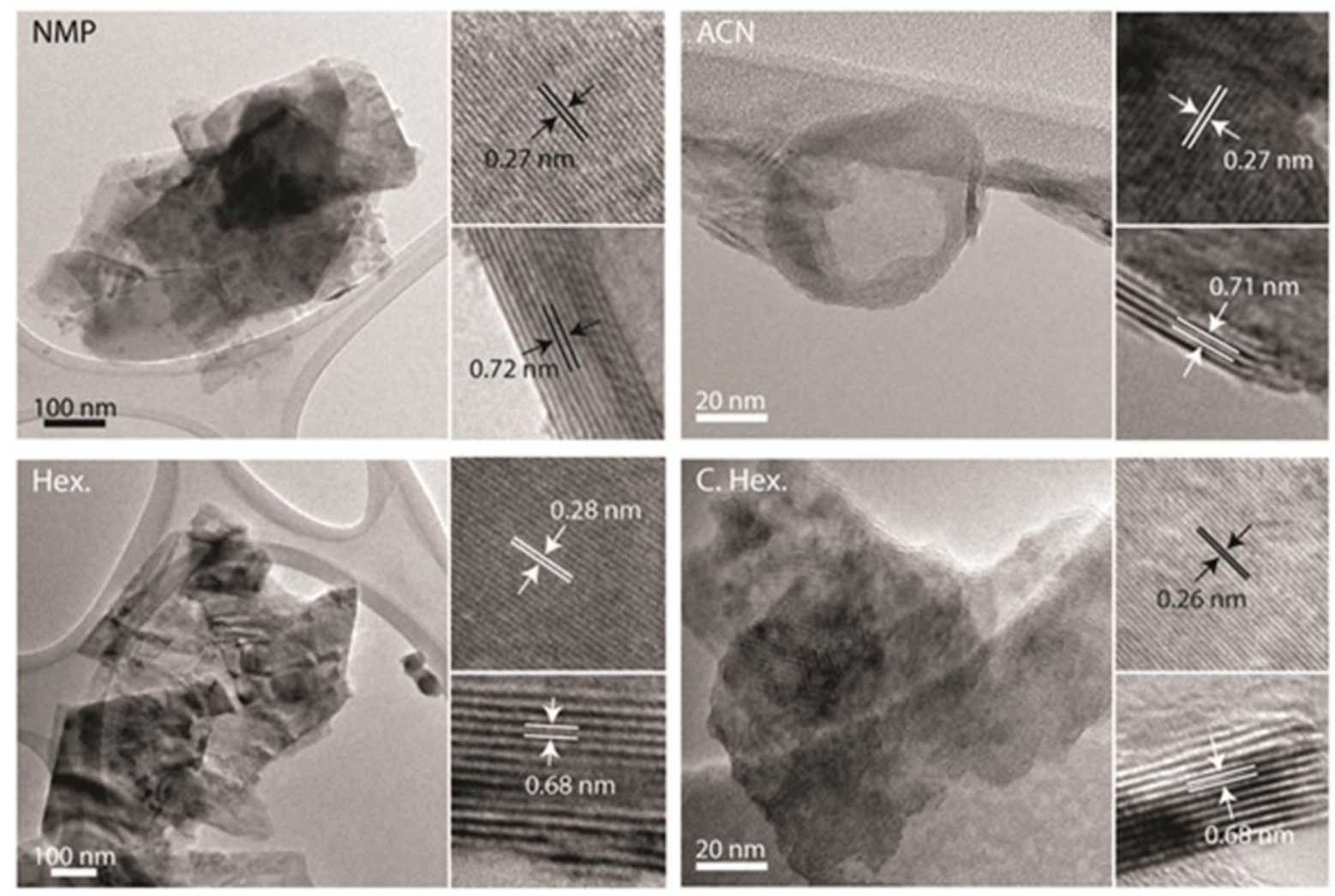

Figure 2: TEM images of the suspensions, illustrating lattice and layer gratings.

the aspect ratio of the lateral dimension to the thickness of the flake, with higher aspect ratios indicating an increased $2 \mathrm{D}$ character. The aspect ratios were in the ranges of 73.331.4 (NMP), 8.6-6.0 (ACN), 7.3-3.6 (Hex.) and 53.3-22.9 (C. Hex.), highlighting that the choice of grinding solvent has a significant influence on the final product. NMP produced the largest aspect ratio for the flakes, which could be attributed to the relatively higher surface tension energy, when compared with the other solvents (see table 1). Studies have suggested that a close match of the surface energy between the solvent and the layered material $\left(40 \mathrm{~mJ} / \mathrm{m}^{2}[28,32]\right)$ leads to the exfoliation of larger flakes with increased aspect ratios.[20, 33] The increased boiling point of the solvent may also have played a role leading to reduced evaporation losses in the case of NMP and increasing the participation of the solvent during the grinding process. The fact, however, that the other investigated solvents, featuring a variety of boiling points and surface tensions, led to negligible $2 \mathrm{D}$ $\mathrm{MoS}_{2}$ concentrations in the ethanolic supernatant suggests that the exfoliation mechanism during the wet grinding process is more complex than anticipated and further studies are necessary to fully elucidate the underlying mechanisms.

Each sample was observed to be well exfoliated in general when investigated under the TEM (Figure 2). Many mono and few layered flakes were identified upon inspection of the edges. Lattice gratings with lattice spacing of $0.27 \mathrm{~nm}\left(\mathrm{~d}_{1-100}\right.$ of $\mathrm{MoS}_{2}$ [ICSD no. 84180]) were easily observed in all samples. The TEM images are in good agreement with the AFM measurements, confirming thicknesses of the flakes and the interlayer spacing of $\sim 0.7 \mathrm{~nm}$, which could be observed at the flakes' edges.

The TEM images in Figure 2 show that exfoliation with NMP produced more angular shaped flakes. The $\mathrm{MoS}_{2}$ layers exfoliated with ACN, Hex. and C. Hex. featured rounder edges and are more random in shape. The Hex. sample was observed to show increased aggregation with clusters of flakes being visible in the TEM images. Flakes exfoliated with C. Hex. tend to have their edges rolled up (see supplementary), indicating that the choice of solvent during the grinding step has influence on the morphology of the exfoliated flakes. Electron diffraction and XRD patterns can be seen in the supplementary.

Optical characterization utilizing UV-vis spectroscopy, Raman spectroscopy and PL measurements were employed to further characterize the suspensions. The exfoliated suspensions were transparent and appear yellow/green in color, where the darkest, and most concentrated, was the ACN sample and the lightest, and least concentrated, was the NMP sample, which appears to be almost colorless (figure 3 inset). Using the method proposed by O'Neill et al. [33]., the concentrations were calculated to be $4.2,9.7,7.8$ and $6.5 \mu \mathrm{g} / \mathrm{ml}$ for NMP, ACN, Hex. and C. Hex., respectively. The concentrations are about two orders of magnitude lower that those reported others, $[6,26,28]$ which typically employ NMP during the sonication step and as a suspension solvent.

The UV-Vis spectra for Q2D $\mathrm{MoS}_{2}$ suspensions are shown in figure 3 . The characteristic absorption bands appear at $660,610,450$ and $395 \mathrm{~nm}$ and are referred to as the A, B, C and D peaks, respectively. [34-36] The A and $\mathrm{B}$ peak arise from direct excitonic transitions at the $\mathrm{K}$ point of the Brillouin zone. [35, 36] The $\mathrm{C}$ and $\mathrm{D}$ peaks can be assigned to the direct excitonic transition of $\mathrm{M}$ point. [5, 35] These characteristic peaks can be observed most clearly in the absorption spectrum obtained for the sample exfoliated with ACN. The D peak is not as conspicuous for the suspensions in Hex. and C. Hex. In the case of NMP these peaks are lacking in the UV-Vis spectra. This can be partly attributed to the lower concentration obtained in comparison to the other 
suspensions.

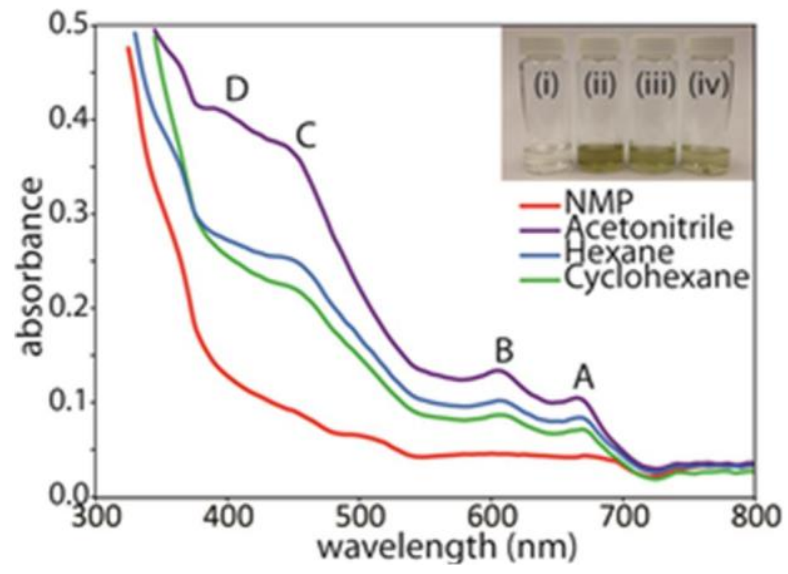

Figure 3: UV-vis spectra of Q2D $\mathrm{MoS}_{2}$ samples exfoliated with grinding in various solvents. Inset: photo of the suspensions ground with (i) NMP, (ii) ACN, (iii) Hex. and (iv) C. Hex.

Raman spectroscopy is routinely utilized to characterized thin layered materials. Figure 4(a) shows the full Raman spectra of the suspensions in the 200 to 2000 $\mathrm{cm}^{-1}$ range. The sharp peak at $520 \mathrm{~cm}^{-1}$ appears due to the silicon substrate.

Two very broad and intense Raman peaks (1360 and $1580 \mathrm{~cm}^{-1}$ ) and broad background scattering throughout the organic fingerprint region (500 to $2000 \mathrm{~cm}^{-1}$ [37]) were observed for the NMP ground $\mathrm{MoS}_{2}$ samples. The two peaks can be allocated to NMP,[38] which indicates that the solvent was not fully removed from the surface, despite heating at $60{ }^{\circ} \mathrm{C}$ and reduced pressure for several hours and redispersing in ethanol. The 300 to $500 \mathrm{~cm}^{-1}$ region in which the main $\mathrm{MoS}_{2}$ signature Raman peak shifts are located is overshadowed by the broad background Raman scattering, leading to comparatively weak Raman signals. The presence of NMP on the surface of the dried flakes is detrimental for the many potential applications of Q2D $\mathrm{MoS}_{2}$, especially for sensing and catalysis, for which the $\mathrm{MoS}_{2}$ surface needs to directly interact with the analyte or educts. Furthermore, the intense signal of NMP could not be removed from the surface is strong evidence that NMP coordinates to the $\mathrm{MoS}_{2}$ surface and cannot simply be seen as just a solvent. In this case, NMP is likely also to alter the electronic properties of $\mathrm{MoS}_{2}$. NMP is a highly polarized material that affects the location of charges within the Q2D material. C. Hex. ground particles also exhibit a broad background scattering throughout the higher wavenumbers, however the intensity is smaller when compared to the NMP sample and no characteristic peaks were observed. Suspensions ground with Hex. and ACN show no broad background or solvent peaks, indicating a nearly solvent free pristine surface.

The $\mathrm{E}_{2 \mathrm{~g}}^{1}$ and $\mathrm{A}_{1 \mathrm{~g}}$ peaks of $\mathrm{MoS}_{2}$ can be observed in all samples (figure 4 (b)). It is well known that Raman spectroscopy can be used to distinguish between bulk and exfoliated 2D material $[3,4,9]$ and can thus be used to assess the quality of the exfoliation. It has been reported that shifts in the $E_{2 g}^{1}\left(383 \mathrm{~cm}^{-1}\right)$ and $A_{1 g}\left(408 \mathrm{~cm}^{-1}\right)$ peaks occur when reducing the number of layers of the $2 \mathrm{D}$ material. $[3,4,35]$ A red shift of the $E_{2 g}^{1}$ peak and a blue shift of the $A_{1 g}$ peak are typically observed for thin layers. This is commonly accompanied by a change in intensity of these peaks, which has also been observed for the here investigated samples. This shift in the wavenumber is associated with thinner flakes obtained for NMP and C. Hex and thicker ones for Hex. and ACN., which are in agreement with the AFM investigations.

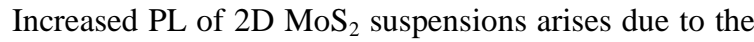
transition from indirect bulk $\mathrm{MoS}_{2}$ to direct band gap few or single layer $\mathrm{MoS}_{2}$. [7, 8, 21, 39] Emission arises from the from the $\mathrm{K}$ point in Brillioun zone $[5,7,34]$ as well as the quantum confinement effect when their lateral dimensions are comparable to those of
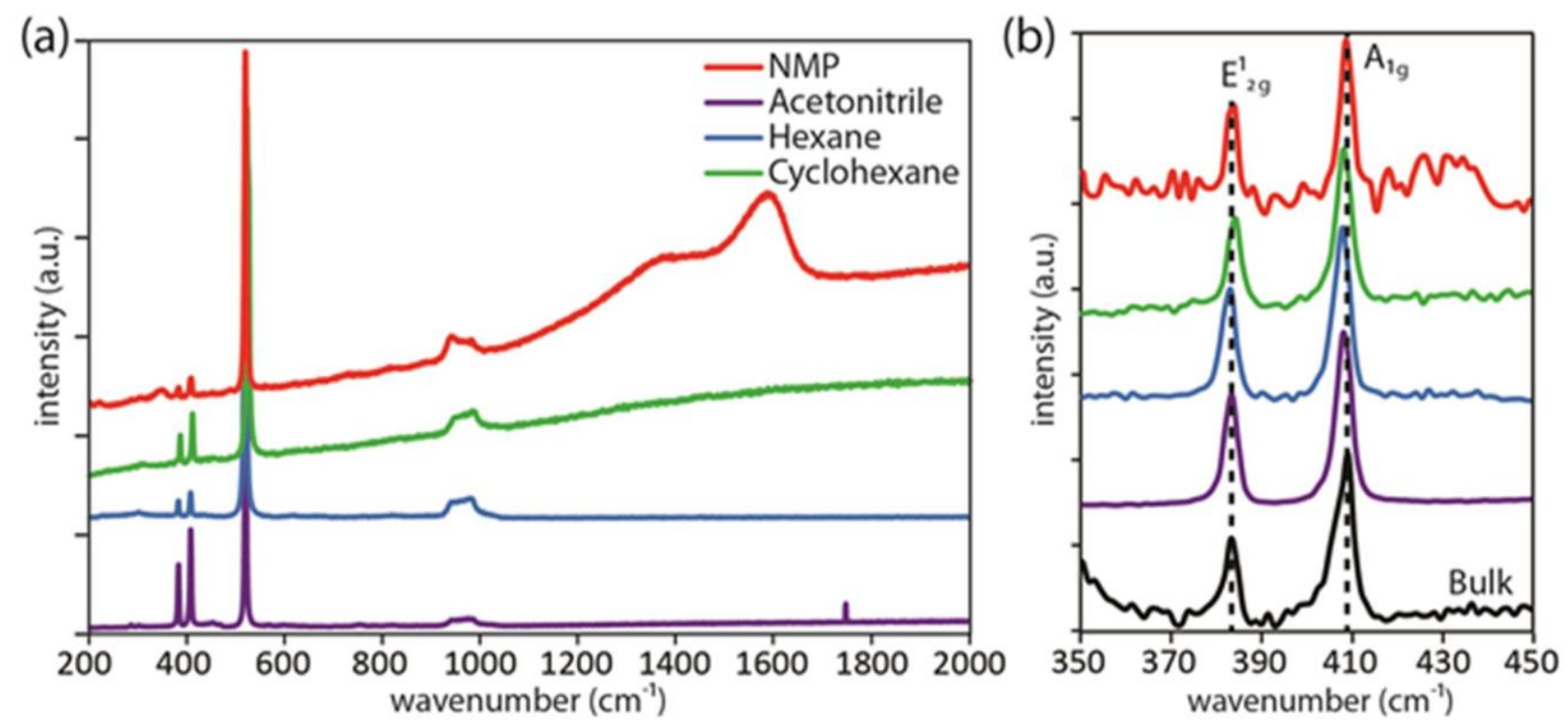

Figure 4: (a) Normalised Raman spectra (485 nm) and (b) enlargement of the $\mathrm{MoS}_{2}$ peaks $\mathrm{E}_{2 \mathrm{~g}}^{1}$ and $\mathrm{A}_{1 \mathrm{~g}}$. 

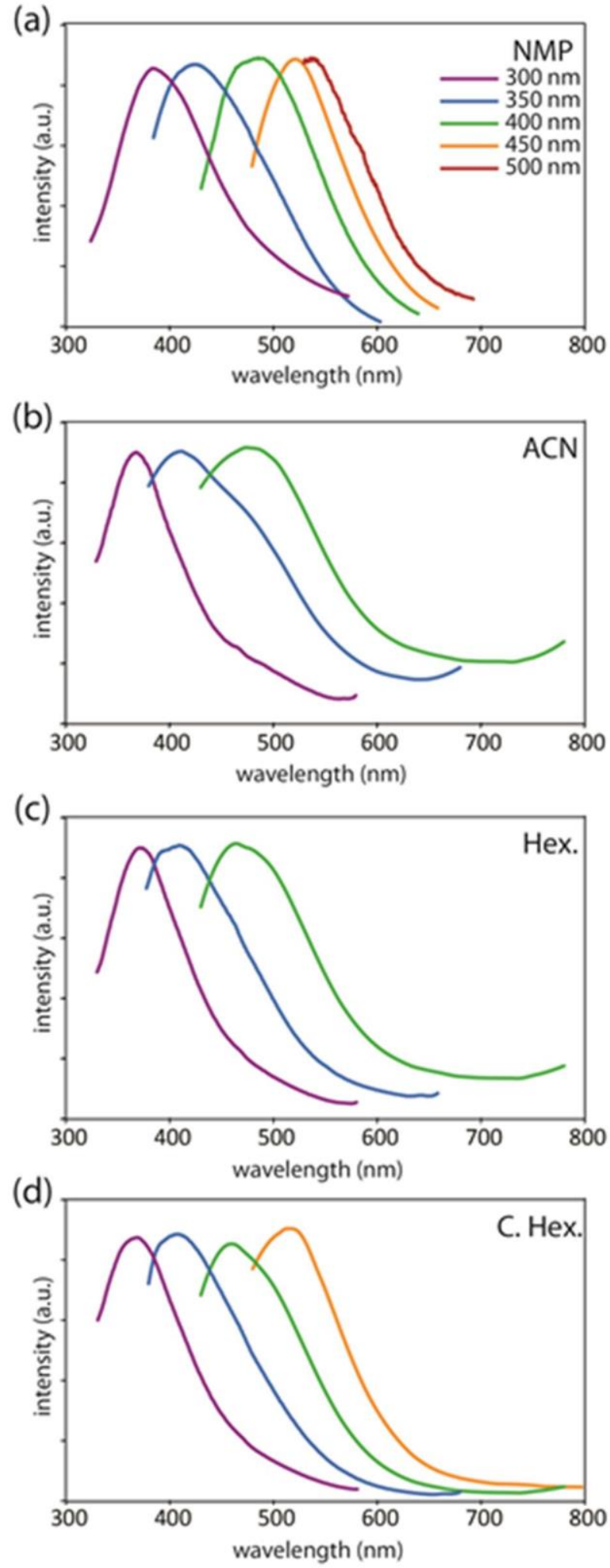

Figure 5: Photoluminescence spectra of the Q2D nanoflake suspensions excited at 300, 350, 400, 450 and $500 \mathrm{~nm}$.

excitons. [10, 14, 15, 21] The PL spectra of each sample excited at various wavelengths $(300,350,400,450$ and $500 \mathrm{~nm}$ ) can be seen in Fig. 5. PL was found for all samples, indicating the presence of direct band gap few layer semiconducting $\mathrm{MoS}_{2}$.

The observed emission peak exhibited red-shifts with increasing excitation wavelengths in all cases. This redshift of the PL signal has been described in the literature and can be rationalized by quantum confinement effects due to the distribution of lateral sizes of the flakes.[15, 35, 39] Strengl et al., have shown that the PL of $\mathrm{MoS}_{2}$ quantum dots shifts with the excitation wavelength allowing to distinguish between quantum dots with different sizes.[39] Applying this concept to our samples, we can expect that low energy photons will excite the larger flakes present in the suspension, leading to a red shift in the PL spectrum, while high energy photons will also excite smaller flakes resulting in the observed blue shift of the PL. Analysing the PL spectra at various excitation wavelengths allows us to infer information on the particle size distribution, which is complementary to the DLS, AFM and TEM analysis. However, since PL occurs exclusively in the thin, direct band gap flakes, it allows us to distinguish between the not desired thicker, indirect bandgap bulk flakes and the sought after thin, direct band gap semiconducting flakes. DLS in unable to distinguish between these two distributions.

Q2D $\mathrm{MoS}_{2}$ flakes ground in NMP show intense PL for excitation wavelengths between 300 and $500 \mathrm{~nm}$, while C. Hex. ground flakes do not feature PL for excitation wavelength above $450 \mathrm{~nm}$. ACN and Hex. ground samples do not feature PL about $400 \mathrm{~nm}$ excitation. This leads to the conclusion that the largest direct band gap Q2D $\mathrm{MoS}_{2}$ flakes are present in the NMP ground sample, whereas smaller flakes are present in the C. Hex., ACN and Hex. showing the smallest size of direct band gap flakes.

(a)

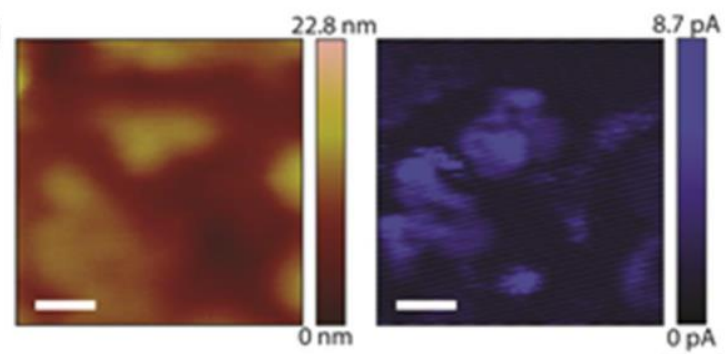

(b)

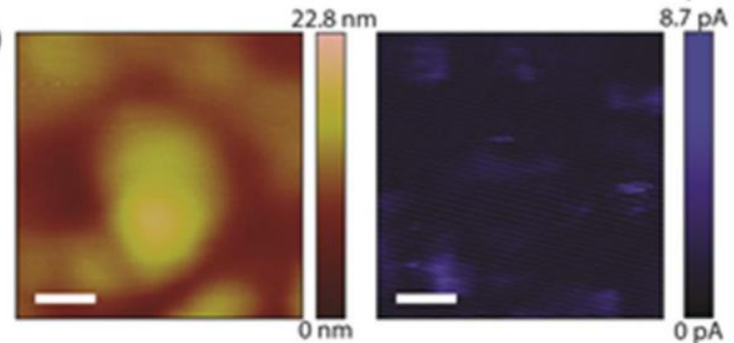

Figure 6: Conductive AFM demonstrating (a) conductive areas (b) and non-conductive areas. The scale bar is $100 \mathrm{~nm}$.

Conductive AFM was performed on the ACN ground nanoflakes to investigate the quality of the samples in terms surface conductivity. Previous work [2, 18] suggests that nanoflakes with defects (loss of sulphur atoms) on the surface edges possess more conductivity along the flake edges when compared to the flake's center. Two different regions are shown in Fig. 6 as an example. It was observed that some nanoflakes were more conductive than others indicating that some flakes possess more defects than others. Furthermore, the higher conductivity regions of the sample were not confined to the edges, indicating that the defects are not limited to the flake edges.

\section{Conclusion}

The grinding assisted exfoliation of $\mathrm{MoS}_{2}$ was investigated using a two-solvent approach. Ten organic solvent were investigated during the grinding phase, which was followed by the removal of the solvent and sonication in ethanol. It was found that the grinding step and the choice of grinding solvent prior to the ultrasound liquid phase exfoliation is playing a crucial role which has been overlooked to date. Just four grinding solvents led to the 
successful exfoliation of Q2D $\mathrm{MoS}_{2}$ nanoflakes in moderate yields. AFM, DLS and PL measurements revealed that the lateral size and thickness of the exfoliated flakes highly dependent on the chosen solvent during the grinding step. NMP was found to produce the highest quality flakes in regards to lateral size and flake thickness. Raman spectroscopy, however, provided strong evidence that NMP remained on the surface of the exfoliated flakes despite the efforts to remove the solvent after the grinding step, suggesting that NMP may be coordinated to the $\mathrm{MoS}_{2}$ surface, prohibiting its removal. This may have detrimental effects on certain applications of Q2D $\mathrm{MoS}_{2}$ that require pristine, clean surfaces such as gas sensing, energy storage and catalysis. ACN was identified as a promising alternative to NMP due to satisfactory exfoliation yields and the complete removal from the $\mathrm{MoS}_{2}$ surface after grinding. The size of the exfoliated flakes was however smaller when compared to NMP ground $\mathrm{MoS}_{2}$. Conductive AFM showed that lattice defects are present in the ground flakes, giving rise to conductivity. The defect density however varied between flakes.

This work highlights the importance of the grinding step during the grinding assisted ultrasound exfoliation of 2D materials which is poorly understood to date. Further research may lead to increased exfoliation yields and larger lateral dimensions of the flakes. The results also highlight that the commonly used exfoliation solvent NMP remains on the surface and may in fact coordinate to the surface. Future work should carefully evaluate, if NMP residues on the Q2D $\mathrm{MoS}_{2}$ surface can interfere with the intended function.

\section{ASSOCIATED CONTENT}

More TEM images, Electron Diffraction and XRD patterns can be found in the supporting information. This material is available free of charge via the Internet at http://pubs.acs.org.

\section{AUTHOR INFORMATION}

\section{Corresponding Author}

* Emails: emily.nguyen@csiro.au and

kourosh.kalantar@rmit.edu.au

\section{Author Contributions}

All authors have given approval to the final version of the manuscript.

\section{Notes \\ † Unpublished data}

\section{ACKNOWLEDGMENT}

The authors acknowledge the facilities, and the scientific and technical assistance, of the Australian Microscopy and Microanalysis Research Facility at the RMIT Microscopy \& Microanalysis Facility (RMMF), at RMIT University and of the Commonwealth Scientific and Industrial Research Organisation (CSIRO), Highett, Victoria, Australia.

\section{ABBREVIATIONS}

ACN, acetonitrile; C. Hex., cyclohexane; Hex. hexane; NMP, N-methyl-2-pyrrolidone; Q2D, quasi two dimensional; MoS2, molybdenum disulphide; AFM, atomic force microscopy; DLS, dynamic light scattering; TEM, transmission electron microscopy; PL, photoluminescence;

\section{REFERENCES}

[1] X. You, et al., "An electrochemical route to $\mathrm{MoS}_{2}$ nanosheets for device applications," Materials Letters, vol. 121, pp. 31-35, 2014.
[2] O. Y. Posudievsky, et al., "Improved dispersant-free liquid exfoliation down to the graphene-like state of solvent-free mechanochemically delaminated bulk $\mathrm{MoS}_{2}, "$ Journal of Materials Chemistry C, vol. 1, pp. 6411-6415, 2013.

[3] H. Li, et al., "From Bulk to Monolayer $\mathrm{MoS}_{2}$ : Evolution of Raman Scattering," Advanced Functional Materials, vol. 22, pp. 1385-1390, 2012.

[4] C. Lee, et al., "Anomalous Lattice Vibrations of Single- and Few-Layer $\mathrm{MoS}_{2}, "$ ACS Nano, vol. 4, pp. 2695-2700, 20102010.

[5] K. F. Mak, et al., "Tightly bound trions in monolayer $\mathrm{MoS}_{2}$," Nat Mater, vol. 12, pp. 207-211, 2013.

[6] G. S. Bang, et al., "Effective Liquid-Phase Exfoliation and Sodium Ion Battery Application of $\mathrm{MoS}_{2}$ Nanosheets," ACS Applied Materials \& Interfaces, vol. 6, pp. 7084-7089, 20142014.

[7] G. Eda, et al., "Photoluminescence from Chemically Exfoliated $\mathrm{MoS}_{2}, "$ Nano Letters, vol. 11, pp. 51115116, 20112011.

[8] A. Sobhani, et al., "Enhancing the photocurrent and photoluminescence of single crystal monolayer $\mathrm{MoS}_{2}$ with resonant plasmonic nanoshells," Applied Physics Letters, vol. 104, pp. -, 2014.

[9] T. Korn, et al., "Low-temperature photocarrier dynamics in monolayer $\mathrm{MoS}_{2}, "$ Applied Physics Letters, vol. 99, pp. -, 2011.

[10] A. Splendiani, et al., "Emerging Photoluminescence in Monolayer $\mathrm{MoS}_{2}$," Nano Letters, vol. 10, pp. 1271-1275, 20102010.

[11] B. Radisavljevic, et al., "Single-layer $\mathrm{MoS}_{2}$ transistors," Nat Nano, vol. 6, pp. 147-150, 2011.

[12] H. Hwang, et al., "MoS 2 Nanoplates Consisting of Disordered Graphene-like Layers for High Rate Lithium Battery Anode Materials," Nano Letters, vol. 11, pp. 4826-4830, 20112011.

[13] K. Zhang, et al., "Unconventional Pore and Defect Generation in Molybdenum Disulfide: Application in High-Rate Lithium-Ion Batteries and the Hydrogen Evolution Reaction," ChemSusChem, 2014.

[14] K. F. Mak, et al., "Atomically Thin $\mathrm{MoS}_{2}$ : A New Direct-Gap Semiconductor," Physical Review Letters, vol. 105, p. 136805, 2010.

[15] J. Z. Ou, et al., "Ion-Driven Photoluminescence Modulation of Quasi-Two-Dimensional $\mathrm{MoS}_{2}$ Nanoflakes for Applications in Biological Systems," Nano Letters, vol. 14, pp. 857-863, 20142014.

[16] N. Perea-López, et al., "CVD-grown monolayered $\mathrm{MoS}_{2}$ as an effective photosensor operating at lowvoltage," 2D Materials, vol. 1, p. 011004, 2014.

[17] U. Halim, et al., "A Rational Design of Cosolvent Exfoliation of Layered Materials by Directly Probing Liquid-Solid Interaction," Nat. Commun., vol. 4, 2013.

[18] S. Park and R. S. Ruoff, "Chemical methods for the production of graphenes," Nat Nano, vol. 4, pp. 217-224, 2004.

[19] C.-J. Shih, et al., "Bi- and trilayer graphene solutions," Nat Nano, vol. 6, pp. 439-445, 2011.

[20] V. Nicolosi, et al., "Liquid Exfoliation of Layered Materials," Science, vol. 340, 20132013.

[21] Q. Tang and Z. Zhou, "Graphene-analogous lowdimensional materials," Progress in Materials Science, vol. 58, pp. 1244-1315, 2013.

[22] K. E. Dungey, et al., "Structural Characterization and Thermal Stability of $\mathrm{MoS}_{2}$ Intercalation Compounds," Chemistry of Materials, vol. 10, pp. 2152-2161, 19981998.

H. S. S. Ramakrishna Matte, et al., "MoS 2 and $\mathrm{WS}_{2}$ Analogues of Graphene," Angewandte Chemie, vol. 122, pp. 4153-4156, 2010. 
[24] J. Heising and M. G. Kanatzidis, "Structure of Restacked $\mathrm{MoS}_{2}$ and $\mathrm{WS}_{2}$ Elucidated by Electron Crystallography," Journal of the American Chemical Society, vol. 121, pp. 638-643, 1999 1999.

[25] Y. Wang, et al., "Electrochemical Control of Photoluminescence in Two-Dimensional $\mathrm{MoS}_{2}$ Nanoflakes," ACS Nano, vol. 7, pp. 10083-10093, 2013.

[26] Y. Yao, et al., "High-Concentration Aqueous Dispersions of $\mathrm{MoS}_{2}, "$ Advanced Functional Materials, vol. 23, pp. 3577-3583, 2013.

[27] K.-G. Zhou, et al., "A Mixed-Solvent Strategy for Efficient Exfoliation of Inorganic Graphene Analogues," Angewandte Chemie International Edition, vol. 50, pp. 10839-10842, 2011.

[28] J. N. Coleman, et al., "Two-Dimensional Nanosheets Produced by Liquid Exfoliation of Layered Materials," Science, vol. 331, pp. 568-571, 20112011.

[29] D. R. Lide, ed., Physical Constants of Organic Compounds. Boca Raton, FL: CRC Press, 2005.

[30] T. J. Wieting and J. L. Verble, "Infrared and Raman Studies of Long-Wavelength Optical Phonons in Hexagonal $\mathrm{MoS}_{2}$," Physical Review B, vol. 3, pp. 4286-4292, 1971.

[31] M. Lotya, et al., "Measuring the lateral size of liquid-exfoliated nanosheets with dynamic light scattering," Nanotechnology, vol. 24, p. 265703, 2013.

[32] S. D. Bergin, et al., "Towards Solutions of SingleWalled Carbon Nanotubes in Common Solvents," Advanced Materials, vol. 20, pp. 1876-1881, 2008.

[33] A. O'Neill, et al., "Preparation of High Concentration Dispersions of Exfoliated $\mathrm{MoS}_{2}$ with Increased Flake Size," Chemistry of Materials, vol. 24, pp. 2414-2421, 20122012.

[34] J. P. Wilcoxon, et al., "Synthesis and optical properties of $\mathrm{MoS}_{2}$ and isomorphous nanoclusters in the quantum confinement regime," Journal of Applied Physics, vol. 81, pp. 7934-7944, 1997.

[35] D. Gopalakrishnan, et al., "MoS 2 Quantum DotInterspersed Exfoliated $\mathrm{MoS}_{2}$ Nanosheets," ACS Nano, vol. 8, pp. 5297-5303, 20142014.

[36] V. Chikan and D. F. Kelley, "Size-Dependent Spectroscopy of $\mathrm{MoS}_{2}$ Nanoclusters," The Journal of Physical Chemistry B, vol. 106, pp. 3794-3804, 20022002.

[37] D. Lin-Vien, et al., The Handbook of Infrared and Raman Characteristic Frequencies of Organic Molecules: Elsevier, 1991.

[38] H. J. Kim, et al., "In situ study on kinetic behavior during asymmetric membrane formation via phase inversion process using Raman spectroscopy," Journal of Applied Polymer Science, vol. 75, pp. 135-141, 2000.

[39] V. Stengl and J. Henych, "Strongly luminescent monolayered $\mathrm{MoS}_{2}$ prepared by effective ultrasound exfoliation," Nanoscale, vol. 5, pp. 3387-3394, 2013. 